\title{
Simulation Modeling and Analysis for improvement of performance measures in a FMS
}

\author{
A. Sreenivasulu ${ }^{1}$, N. Venkatachalapathi ${ }^{2}$ G. Prasanthi ${ }^{3}$ \\ ${ }^{1}$ (Mechanical, VRSEC, Vijayawada, India) \\ ${ }^{2}$ (Mechanical, Annamacharya Institute ofTechnology, Rajampeta,India) \\ ${ }_{3}^{3}$ (Director, Industrial Relations \& Placements and, School of Continuing and Distance Education, JNTUA,
} Ananthapuramu, India)

\begin{abstract}
This paper deals with a simulation study to investigate the effects of various scheduling policies on the performance of a typical flexible manufacturing system (FMS). The scheduling issues considered are part launching, part sequencing at central buffer and tool selection rules on a flexible manufacturing system (FMS) involving tool movement along with part movement policy. The simulation model is developed using $C$ language. This simulation ensured bias-free output after verification, validation steady state analysis. Detailed analysis has been carried out using different scheduling rules. The performance measures considered make span, mean flow time, mean machine utilization and mean tardiness. The results achieved through the simulation have been statistically investigated. The best possible scheduling rules combinations for part launching, tool selection and part sequencing at central buffer have been identified for the selected flexible manufacturing system (FMS).
\end{abstract}

Keywords: FMS; scheduling; simulation; performance measures.

\section{Introduction}

Manufacturing trades operate in a progressively competitive environment. Conventional job shops are designed to manufacture small batches of variety of products. This flexibility is usually off-set by low utilization of the production facilities, long lead times and high in process inventories. On the other hand, fixed sequence transfer lines are typically designed at large volume production, high machine utilization and short lead time. Enhancing the productivity of job shop without sacrificing its flexibility had been a long awaited dream of manufacturing system managers. Emergence of flexible manufacturing system (FMS) is an important development in this direction using the novel concept of flexible automation.

A flexible manufacturing system is a production system in which group of computer numerically controlled (CNC) machines and a material handling system, work collectively under computer control. The decision problems in flexible manufacturing system are classified as design, planning, scheduling and control problems. In the present paper, the focus is on scheduling problems of FMS. The scheduling problems of FMS, in essence, are concerned with the following decisions [1]:

(1) Part launching rules part (Input sequencing of parts in to the system)

(2) Part sequencing rules at central buffer

(3) Tool selection rules.

FMS scheduling methods developed in the previous research found to range from rules of uncomplicated comprehensive algorithms. One of The most significant reviews of scheduling rules in flexible manufacturing system was presented by Gupta et al [2]. Artificial neural network method for solving existent time scheduling and part sequencing was developed by Jones et al. A neural network method creates novel schedule rule when it is necessary. Simulation of FMS is to forecast the performance of each rule in opposition to every performance measures is used [3].

Three different heuristics are used to tackle due-date based job assignment, tool assignment and job sequencing problems in a typical FMS, through a major objective of optimizing total tardiness was prepared by Roh and Kim. They were compared in opposition to each other with simulation experimentation by SIMAN software [4]. An incorporated simulation model based method to determine the scheduling of problems of operations in a Flexible Manufacturing System was presented by Mohamed. Performance measures such as mean lateness, MFT, mean tardiness and mean system utilization were estimated using FORTRAN concerted with the SLAMII software [5].

Simulation based approach to study the reactive scheduling in the stochastic and dynamic environment of FMS was used by Sabuncuoglu and Kizilisik [6]. A heuristic methodology to resolve the problem of machine-loading in the FMS was developed by Goswami and Tiwari. Machines are capable to carry out a variety of operations on numerous alternative machines in this FMS environment [7]. 
A simulation based approach was developed by Joseph and Sridharan to expect the effect of priority scheduling rules with controlling of job selection and selection of tool decision of Flexible Manufacturing System and considered two different scenarios primary one, every resources tool transporters (TT), machines and part transporters (AGV) are expected to be always accessible without failure next one these available resources are with break downs, these two different scenarios are used test the simulation models. Various combinations of scheduling rules are integrated and measured an every scheduling rule the performance. Performance measures are mean tool waiting time for tardy part, mean flow time and the best possible combinations of scheduling rules recognized [8,9].

Task based AGV scheduling in a Flexible Manufacturing System by means of non-traditional optimization techniques were considered by Udhaya kumar and Kumanan. The study of scheduling of Flexible Manufacturing System has created a lot of interest and several algorithms of mathematical have been formed for solving the problem of FMS scheduling [10]. Research dealt with definite FMS configurations and focused on distinct objective optimization. A simulation model for optimization of different job input sequence in a FMS was developed by Howe and David [11]. The simulation based model was developed by using user-interface software (Flexsim) that permits different job and process time data fitted out in an Excel sheet to be introduced into the simulation based model. The module of optimization of simulation software is run dissimilar orders of different job input to find out the maximum total slack.

From the literature it is evident that tool sharing and different part launching rules optimizes the performance measures $[12,13,14,15]$. However, not much concentration has been given to study the effect of part sequencing at central buffer on system performance. The possibility of part sequencing rules at central buffer in a FMS environment can be assessed by formulating and evaluating the part sequencing rules at central buffer. Also, the study of part sequencing rules at central buffer would guide to understanding the performance in a completely automated system. The proposed research concentrates on the effect of different combinations of part launching, part sequencing rules at central buffer and tool selection rules in an FMS. FMS physical configuration for the purpose of the simulation study, a typical FMS configuration is selected. The details layout of the FMS configuration mentioned in Table.1 is illustrated in Fig. 1.Physical configuration consists of one load/unload (L/UL) station, four machine groups, each group consists of two identical machines, totally 8 machines (M1, M2, M3, M4, M5, M6, M7, M8). Two identical AGVs provide part-handling services between load - unload station, workstations and central buffer. Four tool trans-porters are used to transport tools between machines and tool stores, also to passage tools between machines.

Table .1. Physical configuration of the simulation model.

\begin{tabular}{lc} 
Part Types Considered for Production & 20 \\
\hline Machines & \\
Number of machine groups & 4 \\
Distance between the machine groups & $8 \mathrm{~m}$ \\
Number of machines in each group & 2 \\
Distance between the machines in & $8 \mathrm{~m}$ \\
each group & \\
Total number of machines & $4 \times 2=8$ \\
The tool magazine capacity & 20 \\
AGVs for part transport & 2 \\
AGV velocity & $3 \mathrm{~m} / \mathrm{s}$ \\
L/UL station & 1 \\
TTs & 4 \\
TT velocity & $3 \mathrm{~m} / \mathrm{s}$ \\
Tool stores & 4 \\
Buffer capacity on machine & \\
Input buffer & 1 \\
Output buffer & 1 \\
Central buffer capacity & 20
\end{tabular}




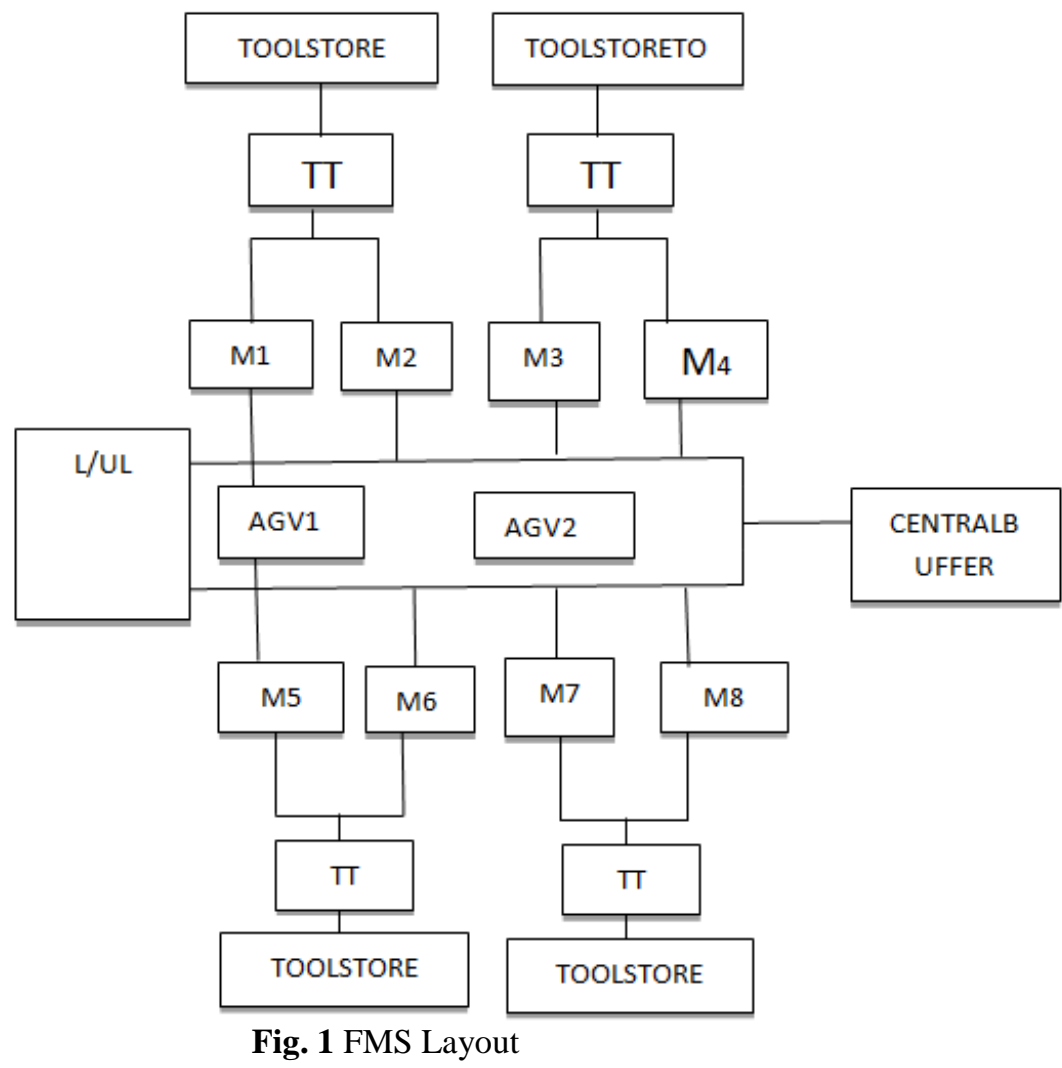

An AGV can travel in any direction and it can carry only 1(one) part at a time. There are 20 part types in this model. Each type has a different process order. For each operation, different type of cutting tool and the processing time at each machine is needed. Process order involves 2-4 major operations uniformly distributed and each major operation contains minor operations uniformly distributed between 5 and 10. Cutting tool required for each operation at each machine group is generated according to uniform distribution. A different due date is defined for each part. Inter arrival time of order for processing in the system follows exponential distribution with a mean of $65 \mathrm{~min}$. An order can be for anyone of the 20 part types with an equal possibility. The processing sequence for each part type is determined, in such a way that no machine group is visited more than one time.

\section{Operational Configuration}

The primary situation in the simulation is expected to be unfilled and idle with the initial job order arrival event scheduled to happen at time zero. The job order may fit in to any one of the 20 different job types. Subsequently, the job associated with the job order arrived and job features are identified, i.e. the job order, operations and their processing times, essential tools for every operation for processing the job. The raw part is then loaded on to AGV and is ready to be launched into the system. Jobs are laden according to part selection rules. The job is then released to the machine for the primarily operation if the input buffer of that specific machine is free and also if someone of the 2 (two) AGVs are idle. If the input buffer of the machines is involved, the parts will be dropped at the central buffer from which they will be picked up later based on part selection rules. Upon the end of part transfer, an idle AGV halts at the end point machine or central buffer or $\mathrm{L} / \mathrm{UL}$ station. The machine is laden with the job that is waiting at the input buffer of the machine for processing. If the necessary tool is available on the machine, the machine starts processing the job. If not, the machine places a call for tool. The tool transporter (TT) joins the call. Tools required for processing a job, inaccessible at a machine are moved from alternative machine or tool store. If there are more than one tool requests at a specific time, then a tool selection rule is used for choosing the request to be met. Hence, the tool is moved to the machine. Subsequently the machine finishes processing every job of the minor operations, the job is released to the output buffer of the machine if output buffer of the machine is an empty; or else, the machine is jammed and an AGV call is engaged to take away the job from output buffer. If AGV is available, it picks up the job. If each one of the major operations of the job are over, the job will be conveyed to L/UL station where the completed job is delivered from AGV along with performance measure is calculated. Otherwise, the part is transported to the machine for the succeeding major operation, if the input buffer of the required machine is blank; or else, the AGV delivers the part at the central buffer. 


\subsection{Assumptions for FMS simulation models}

i. All machines in the simulation based model are initially idle.

ii. At simulation time zero, $\mathrm{AGV}$ is expected to be available at $\mathrm{L} / \mathrm{UL}$ station.

iii. Set up time of an operation is included in the processing time of a part.

iv. At a time, each machine can handle only single operation at most.

v. Without any failure AGV and machines are assumed to be operational.

vi. The order of operation for each part is fixed.

vii. Two identical tools are available in the simulation model.

viii. Tool failures are zero.

\section{Development Of Simulation Models}

In the present work, discrete event simulation models are developed for the operation of the FMS. The simulation models are developed using $\mathrm{C}$ language and run on a Personal computer with Intel I5 processor. $\mathrm{C}$ programming language has a greater programming flexibility than certain simulation package.

An Algorithm of the logic of simulation models is shown below.

1. Starts the simulation based system by executing simulation logic.

2. Read the necessary input data from input file.

3. Initialize the input variables part order, machine array and machine group.

4. Determines the subsequent event from event list.

5. Call the routine event to carry out the present assigned event.

6. Perform the allocated event, update the event, and generate the upcoming activities.

7. Repeat the steps 3 and 6 until event list is empty.

8. If (event list $=\mathrm{empty} / \mathrm{no}$ event requests to execute), prints performance measures of simulation based model values in to the output files.

9. Exit.

\subsection{Part launching rules}

\subsubsection{First come first served (FCFS)}

In this scheduling rule, parts are launched into simulation system based on sequence of their arrival.

3.3.2. Shortest processing time (SPT)

In this scheduling rule, parts are launched into simulation system based on the order of their processing times.

3.3.3. Earliest due date (EDD)

In this scheduling rule, parts are lunched into simulation system based on the order of their longest processing times.

\subsection{Part sequencing rules at central buffer}

3.4.1. First come first served

AGV picks parts based on sequence of their arrival at the central buffer.

3.4.2. Shortest processing time

AGV picks parts according to the processing time for the next major operation with the part having the smallest value for the operation time taken first.

3.4.3. Earliest due date

$\mathrm{AGV}$ picks parts according to the EDD.

\subsection{Tool request selection rules}

3.5.1. First come first served

Tools are issued to the parts based on the tool requests, with the part that has placed the request first given priority.

3.5.2. Shortest imminent operation (SIO)

Tools are issued to the part that has the SIO time.

3.5.3. Earliest due date

Tools are issued to the part that has the EDD. Due date setting. Due date for an order is computed by total work content method.

Due date $=$ arrival time $+\mathrm{K} *$ processing time of the order. where $\mathrm{K}$ is the due-date tightness factor or flow factor. In the experiments, $\mathrm{K}$ is set equal to 3,5 .

\subsection{Output}

The compilation and consolidating the output of the simulation models to present results are shown in this module such as make span, mean flow time, mean machine utilization, mean tardiness. These performance measures are defined as follows: 


\subsubsection{Make span}

Make span: It is the completion time of all parts i.e., it is the completion time of the last operation of the last part.

3.6.2. Mean Flow time

Mean Flow time: It is defined as the average time a part spends in the system.

Flow time $(\mathrm{Fi})=\mathrm{Ci}-\mathrm{Ai}$

Where $\mathrm{Ci}=$ Completion Time of part $\mathrm{i}$

$\mathrm{Ai}=$ Arrival Time of part $\mathrm{i}$

Mean flow time $=(1 / n) \sum$ Fi where $\mathrm{i}=1$ to $\mathrm{n}(\mathrm{n}=$ number of parts completed $)$.

\subsubsection{Mean tardiness}

Mean tardiness: It is the average tardiness of a part.

Tardiness is the difference between the completion time of a job and its due date.

Lateness of part i, $\mathrm{Li}=\mathrm{Ci}-\mathrm{Di}$

Tardiness of part $\mathrm{i}, \mathrm{Ti}=\operatorname{Max}(0, \mathrm{Li})$

3.6.4. Mean machine utilization

Mean machine utilization: It is the average utilization of machines in the system over the simulation period.

\subsection{Verification and validation of simulation models}

The following steps are followed to make sure the correctness of a conceptual FMS.

- Debugging.

- Checking the internal logic.

- Comparing the model output with manual simulation data.

- Running the model.

\section{Experimentation}

The discrete event simulation model developed for the selected FMS experiments was used. The three scheduling rules used for part launching decision and three rules for part sequencing at central buffer and three for tool request selection rules give rise to 27 experiments. 100 replications were made for each experiment. The initial stage in simulation experimentation was the identification of steady state, the moving average method suggested by Law and Kelton [13] was used for this purpose. The moving average plots for the performance measure indicate when the system reaches steady state at the completion of 1000 parts. Hence, in the simulation experiments, the simulation is continued till the completion of 1500 parts. While evaluating the performance measures, the results for the first 1000 parts are not considered and the performance measures are calculated using the results for the remaining 500 parts.

\section{Results And Discussion}

The problem identified in the present investigation involves the analysis of various tool decision rules, part sequencing rules at central buffer and part launching rules. The simulation results are shown in Tables 2 to 5 exhibit variations. These variations could have been caused by the following factors either independently or due to two ways or three ways interactions among them.

Table 2.Make span Results

\begin{tabular}{|c|c|c|c|c|}
\hline \multirow[t]{2}{*}{ Part Launching Rules } & \multirow{2}{*}{$\begin{array}{l}\text { Scheduling Rules To Pick } \\
\text { Part From Central Buffer }\end{array}$} & \multicolumn{3}{|c|}{ Tool Request Selection Rules } \\
\hline & & FCFS & \$10 & EDD \\
\hline \multirow{3}{*}{ FCFS } & FCFS & 16373 & 17241 & $13792^{*}$ \\
\hline & SPT & 14003 & 15934 & 15819 \\
\hline & EDD & 14495 & 14906 & 13971 \\
\hline \multirow{3}{*}{ SPT } & FCFS & 28050 & 27917 & 29402 \\
\hline & SPT & 29727 & 28557 & 28447 \\
\hline & EDD & 27134 & 26778 & 27379 \\
\hline \multirow[t]{3}{*}{ EDD } & FCFS & 28713 & 29163 & 27251 \\
\hline & SPT & 27923 & 28184 & 28131 \\
\hline & EDD & 27620 & 29077 & 28805 \\
\hline
\end{tabular}

*indicates the best combination of scheduling rule

Make span: From Table.1, it is found that part launching rules, part sequencing rules at central buffer and tool request selection rules have significant impact on this performance measure. It is clearly evident that the part launching rule, First come first served (FCFS) rule gives good results when compared with other part launching rules. The combination of FCFS (part launching rule) - FCFS (part sequencing at central buffer) - EDD (tool request selection rule) gives the best value for make span. 
Table.3. Mean Flow Time Results

\begin{tabular}{|c|c|c|c|c|}
\hline \multirow[t]{2}{*}{$\begin{array}{l}\text { Part Launching } \\
\text { Rules }\end{array}$} & \multirow[t]{2}{*}{$\begin{array}{l}\text { Scheduling Rules To Pick } \\
\text { Part From Central Buffer }\end{array}$} & \multicolumn{3}{|c|}{ Tool Request Selection Rules } \\
\hline & & FCFS & SIO & EDD \\
\hline \multirow{3}{*}{ FCFS } & FCFS & 81 & 86 & 70 \\
\hline & SPT & 70 & 79 & 79 \\
\hline & EDD & 72 & 74 & $69 *$ \\
\hline \multirow{3}{*}{ SPT } & FCFS & 140 & 139 & 147 \\
\hline & SPT & 148 & 142 & 142 \\
\hline & EDD & 135 & 133 & 136 \\
\hline \multirow{3}{*}{ EDD } & FCFS & 143 & 145 & 136 \\
\hline & SPT & 139 & 140 & 140 \\
\hline & EDD & 138 & 145 & 144 \\
\hline
\end{tabular}

*indicates the best combination of scheduling rule

Mean Flow Time: From Table.3, it is found that FCFS rule provides minimum mean flow time when compared with other part launching rules. The combination of FCFS (part launching rule) - EDD (part sequencing rule at central buffer) - EDD (tool request selection rule) provides the smallest value for mean flow time.

Table. 4. Mean Tardiness Results

\begin{tabular}{|c|c|c|c|c|}
\hline Part Launching Rules & \multirow{2}{*}{$\begin{array}{c}\text { Scheduling Rules To Pick } \\
\text { Part From Central Buffer }\end{array}$} & \multicolumn{3}{|c|}{ Tool Request Selection Rules } \\
\cline { 2 - 5 } & FCFS & 24 & SPT & 24 \\
\hline \multirow{3}{*}{ FCFS } & SPT & 23 & 23 & 23 \\
\cline { 2 - 5 } & EDD & 23 & 23 & 24 \\
\cline { 2 - 5 } & FCFS & 23 & 23 & 24 \\
\hline \multirow{3}{*}{ SPT } & SPT & 24 & 23 & 24 \\
\cline { 2 - 5 } & EDD & 23 & $22 *$ & 23 \\
\cline { 2 - 5 } & FCFS & 23 & 23 & 23 \\
\cline { 2 - 5 } & SPT & 23 & 23 & 23 \\
\hline
\end{tabular}

*indicates the best combination of scheduling rule

Mean Tardiness: From Table. 4, it is found that part launching rules as well as part sequencing at central buffer and tool request selection rules have considerable impact on the performance measure. The best combination of rules is SPT (part launching rule) - EDD (part sequencing rule at central buffer) - SPT (tool request selection rule) gives minimum mean tardiness.

Table. 5. Mean Machine Utilization Results

\begin{tabular}{|c|c|c|c|c|}
\hline \multirow{2}{*}{ Part Launching Rules } & \multirow{2}{*}{$\begin{array}{c}\text { Scheduling Rules To Pick Part } \\
\text { From Central Buffer }\end{array}$} & FCFS & SPT & EDD \\
\cline { 2 - 5 } & FCFS & 98 & 97.5 & 97.5 \\
\cline { 2 - 5 } & SPT & 97.5 & 98 & 97.5 \\
\cline { 2 - 5 } & EDD & 97.5 & 98 & $98^{*}$ \\
\cline { 2 - 5 } & FCFS & 97.1 & 97.5 & 97 \\
\cline { 2 - 5 } & SPT & 97.5 & 97.5 & 97.5 \\
\cline { 2 - 5 } & EDD & 97.5 & 97.5 & 97 \\
\hline \multirow{3}{*}{ EDT } & FCFS & 97.5 & 97 & 97 \\
\cline { 2 - 5 } & SPT & 97.5 & 97 \\
\cline { 2 - 5 } & EDD & & & 97 \\
\hline
\end{tabular}

*indicates the best combination of scheduling rule

Mean Machine Utilization: The results presented in Table 5 reveal that the machine utilizations do not differ very much for the various combinations of scheduling rules. However, the combination of rules, FCFS (part launching rule) - EDD (part sequencing rule at central buffer) - EDD (Tool request selection rule) provides better values for mean utilization of machines.

\section{Conclusion}

In the current work, a simulation analysis conducted to examine the effect of scheduling policies on the performance of a typical FMS. The simulation has been passed through multi-level validation checks. The output obtained has been suitably subjected to steady state analysis to make sure that further investigations are bias free. The results on detailed analysis revealed a number of interesting facts about the flexible manufacturing system considered. Changes in operating policies signified by scheduling rules influence the system 
performance characterized by various performance measures differently, In general, the policies for part launching, tool request selection and part sequencing at central buffer significantly affect the performance of the system. Clear understanding of the dynamics in the system indicated by interaction effects helps considerably to establish the best operational conditions. These facts have been established in a restricted sense in the present simulation study using the limited number of situations measured. However, the results of this simulation study have provided considerable encouragement to study the system more in detail.

Simulation analysis can be carried out for more scheduling for part launching, part sequencing at central buffer and tool request selection rules.

\section{Acknowledgments}

The authors are grateful to the anonymous referees for their constructive comments, which have helped to improve the paper over its earlier version

\section{References}

[1]. K. E. Stecke, Design, planning, scheduling and control problems in flexible manufacturing system, An Oper. Res. 3 (1985) 3-12.

[2]. Y. P. Gupta, M. C. Gupta and C. R. Bector, A review of scheduling rules in FMS, ICIM 2(6) (1989) $356-377$.

[3]. A. Jones, L. Rabelo and Y. Yuehwern, A hybrid approach for real-time sequencing and scheduling, Int. J. Comput. Integr. Manuf. 8(2) (1995) 145-154

[4]. H.-K. Roh and Y.-D.Kim, Due-date-based loading and scheduling methods for a flexible manufacturing system with an automatic tool transporter, Int. J. Produc. Res. 35 (1997) 2989-3003.

[5]. N. S. Mohamed, Operations planning and scheduling problems in an FMS: An inte-grated approach, Comput. Indust. Eng. 35(3-4) (1998) 443-446.

[6]. I. Sabuncuoglu and O. B. Kizilisik, Reactive scheduling in a dynamic and stochastic FMS environment, Int. J. Produc. Res. 41(17) (2003) 4211-4231.

[7]. M. Goswami and M. K. Tiwari, A reallocation-based heuristic to solve a machine loading problem with material handling constraint in a flexible manufacturing system, International Journal of Production Research, 44(3) (2006) 569-588.

[8]. N. Suresh Kumar and R. Sridharan, Simulation modelling and analysis of part and tool flow control decisions in a FMS, Robotics and computer-integrated manufacturing 25 (2009) 829-839.

[9]. A.Sreenivasulu, N.Venkatachalapathi and G.Prasanthi, Modelling and analysis for minimization of mean flow time in FMS Simulation, Journal of Advanced manufacturing systems, 14(4) (2015) 235-245.

[10]. P. Udhayakumar and S. Kumanan, Task scheduling of AGV in FMS using nontraditional optimization techniques, International Journal of Simulation Modeling. 9(1) (2010) 28-39.

[11]. H. C. Cheng and D. Yinkaichan, Simulation optimization of part input sequence in a flexible manufacturing system, in Proc. Winter Simulation Conference (2011), pp. 2374-2382.

[12]. S. Garapati and H. P. Wang, Decision support system for tool management in FMS, in IEEE International Conference Computer Integrated Manufacturing, Troy, New york (1998), pp. 53-61.

[13]. A. M. Law and W. D. Kelton, Simulation Modeling and Analysis, 3rd edn. (McGraw-Hill Inc., New Delhi, 2000).

[14]. N. Buyurgan, C. Saygin and S. EnginKilic, Tool allocation in FMS with tool alter-natives, Robotics and computer-integrated manufacturing. 20 (2004) 341-349.

[15]. N.Suresh Kumar, R.Sridharan, simulation modeling and analysis of tool sharing and part scheduling decisions in single-stage multi machine flexible manufacturing systems, Robotics and computer-integrated manufacturing.23 (2007) 361-370 